\title{
Situation, Figuration und Gewalt
}

Versuch eines gewaltsoziologischen Dialoges zwischen Randall Collins und Norbert Elias am Beispiel sexueller Kriegsgewalt

\author{
Johannes Ebner • Marion Stopfinger
}

(C) Der/die Autor(en) 2020

Zusammenfassung In diesem Beitrag werden zwei in der soziologischen Gewaltforschung etablierte Ansätze - die mikrosoziologisch-situationistische Gewalttheorie von Randall Collins und die figurations- bzw. prozesssoziologische Perspektive von Norbert Elias - auf ihre Eignung für die Analyse von sexueller Kriegsgewalt überprüft. Nach einer kurzen Diskussion des Forschungsstandes zu sexueller Kriegsgewalt wird dieses Thema einmal mit Collins und einmal mit Elias beleuchtet. Danach werden die beiden Zugänge einander gegenübergestellt, um Unterschiede und Gemeinsamkeiten herauszuarbeiten. Darauf aufbauend wird versucht, die Fruchtbarkeit eines ,pragmatischen Dialoges“ zwischen einem mikro- und einem figurationssoziologisch inspirierten Ansatz auszuloten. Abschließend wird diskutiert, welche Folgerungen sich daraus für die Forschung zu sexueller Kriegsgewalt ergeben.

Schlüsselwörter Randall Collins · Norbert Elias · Gewaltforschung · Figurationssoziologie $\cdot$ Sexuelle Gewalt im Krieg

\footnotetext{
J. Ebner $(\bowtie)$

Institut für interdisziplinäre Konflikt- und Gewaltforschung (IKG), Universität Bielefeld, Universitätsstraße 25, 33615 Bielefeld, Deutschland

E-Mail: johannes.ebner@uni-bielefeld.de 


\section{Situation, figuration, and violence}

Attempting a dialogue between Randall Collins and Norbert Elias considering as example sexual violence in war

Abstract In this paper, two approaches established in sociological violence research - Randall Collins' micro-sociological theory of violence and Norbert Elias' figuration- and process-sociological perspective-are examined for their suitability for the analysis of sexual violence in war. After a brief discussion of the current state of research on sexual violence in war, this topic will be examined once with Collins and once with Elias. The two approaches are then juxtaposed in order to highlight differences and similarities. Building on this, the fruitfulness of a "pragmatic dialogue" between a micro- and a figuration-sociologically inspired approach will be explored. The concluding section discusses the implications for research on sexual violence in war.

Keywords Randall Collins · Norbert Elias · Sociology of violence $\cdot$ Figuration research $\cdot$ Sexual violence in war

\section{Einleitung}

In diesem Beitrag werden einige theoretische und methodische Streitfragen der soziologischen Gewaltforschung am Beispiel der sexuellen Gewalt im Krieg diskutiert. $\mathrm{Zu}$ diesem Zweck werden zwei in der soziologischen Gewaltforschung etablierte Ansätze - Randall Collins' mikrosoziologisch-situationistische Gewalttheorie und Norbert Elias' figurations- bzw. prozesssoziologische Perspektive - auf ihre Eignung für die Analyse von sexueller Kriegsgewalt überprüft. ${ }^{1}$

Unsere Argumentation erfolgt in fünf Schritten: Zunächst werden die unseres Erachtens zentralen Mängel der vorliegenden Literatur zum Thema sexuelle Kriegsgewalt skizziert. Danach folgt unsere Vorgehensweise dem herkömmlichen Muster der analytischen Trennung von (mikro-)situativer und (makro-)struktureller Gewalt, indem wir sexuelle Kriegsgewalt monoperspektivisch einmal mit Collins und einmal mit Elias beleuchten. Im dritten Schritt werden die beiden Zugänge einander gegenübergestellt, um Unterschiede und Gemeinsamkeiten herauszuarbeiten. Darauf aufbauend versuchen wir, die Fruchtbarkeit eines ,pragmatischen Dialoges“ zwischen einem mikro- und einem figurationssoziologisch inspirierten Ansatz auszuloten. Zu diesem Zweck lassen wir die beiden Ansätze gegeneinander laufen, wobei die Konfrontation der Zuspitzung beider Positionen und damit der gedanklichen Schärfung dient. Abschließend wird diskutiert, welche forschungsrelevanten Aspekte zum Thema sexuelle Kriegsgewalt sich aus der Gegenüberstellung der beiden Ansätze ergeben.

\footnotetext{
1 Dieser Aufsatz ist die überarbeitete Version eines Vortrages, den wir anlässlich der Tagung Streitfragen der soziologischen Gewaltforschung (Aachen, 17.-18.11.2017) gehalten haben. Für Anregungen und kritische Kommentare danken wir den dortigen Diskussionsteilnehmer*innen, den Gutachter*innen dieses Beitrages sowie Sabine A. Haring, Jörg Hüttermann und Helmut Kuzmics.
} 
Die Wahl von Collins und Elias hat mehrere Gründe. Beide Euvres bieten vielversprechende Anregungen für die Untersuchung von sexueller Kriegsgewalt. Zudem gibt es sowohl innerhalb des Collins- als auch des Elias-Lagers Zweifel am Ertrag monoparadigmatischer Erklärungen (Ebner 2018; Hoebel und Malthaner 2019; Hoebel und Knöbl 2019; Hüttermann und Ebner 2020). Allerdings hat die Emotionalität der Debatte (Collins 2007, 2011b, 2014; Mennell 2007; Wouters 2007; Wouters und Mennell 2015) bisher eine ernsthafte Auseinandersetzung mit den Einwänden der jeweiligen Gegenposition verhindert. Die Mehrzahl der empirischen Arbeiten beider Forschungstraditionen gibt sich ungeachtet der vorgebrachten Einwände mit der Anwendung einzelner Konzepte zufrieden, ohne diese theoretisch weiterzuentwickeln oder deren Unzulänglichkeiten, Grenzen und Widersprüche zu explizieren (Hoebel und Malthaner 2019, S. 4 ff.). Im Folgenden wollen wir ausloten, ob es sich lohnt, die beiden Soziologien miteinander in Dialog zu bringen - sowohl für beide Perspektiven als auch für die Konzeption von sexueller Kriegsgewalt. Wir hoffen, dass die hier gewählte Form des Dialoges (Selbst-)Erkenntnisse zutage fördern vermag, die über das bloße Anführen interner oder externer kritischer Einwände hinausgehen. Damit reiht sich dieser Aufsatz zum einen in eine Fülle rezenter Diskussionsbeiträge zur erneuten Weiterentwicklung der soziologischen Gewaltforschung ein. Zum anderen versucht er, einen Beitrag zur Re-Konzeption und Versachlichung der Forschung zu sexueller Kriegsgewalt zu leisten.

\section{Schwerpunkte der vorliegenden Literatur zu sexueller Kriegsgewalt}

Seit das Problemfeld sexuelle Kriegsgewalt - zunächst im Rahmen der Frauenbewegung der 1970er Jahre und dann zur Zeit der kriegerischen Konflikte der 1990er Jahre in Jugoslawien und Ruanda - in den Fokus verschiedener Wissenschaftsdisziplinen geraten ist, zeichnet sich die Debatte durch mehrere Schwerpunkte aus.

Die augenscheinlichste, wenngleich nicht überraschendste, Eigentümlichkeit des derzeitigen öffentlichen und wissenschaftlichen Diskurses ist dessen Normativität. Dies ist in erster Linie darauf zurückzuführen, dass er auf dem feministischen Erbe der 1970er Jahre (insbesondere auf Susan Brownmillers (1980 [1975]) Against our Will) basiert, aus dessen Perspektive die Verdammung von sexueller Gewalt zentral für deren Prävention ist. Da verschiedene feministische Perspektiven ${ }^{2}$ bis heute maßgeblichen Einfluss auf die öffentliche und wissenschaftliche Auseinandersetzung ausüben, liegt der Fokus nach wie vor überwiegend auf Opferperspektive und -schutz sowie auf der Verurteilung und Ächtung der Täter*innen. Erst im letzten Jahrzehnt sind Bemühungen zu beobachten, auch die Perspektive der Täter*innen einzubeziehen und das Verstehen des Phänomens in den Vordergrund der (sozial-)wissenschaftlichen Betrachtung zu rücken (Stopfinger 2016).

Eine zweite Wesensart des Diskurses, die mit dessen normativer Ausrichtung zusammenhängt, ist der umfassende Generalisierungsanspruch. Sowohl feministisch-sozialkonstruktivistische Ansätze, die sexuelle Gewalt - in Friedens- wie in Kriegszeiten - pauschal auf vorherrschende Männlichkeitsnormen zurückführen, als

\footnotetext{
2 Für eine differenziertere Darstellung siehe Skjelsbæk (2001, S. 215) oder Goldstein (2001, S. 38 f.).
} 
auch feministisch-biologistisch argumentierende Ansätze, die sexuelle Gewalt auf männliche Aggressivität und Triebhaftigkeit reduzieren, können Unterschiede und Abweichungen in Art, Ausmaß und Intensität der (sexuellen) Gewaltausübung nicht erklären. Sie sind blind für jede Art der Differenzierung - ob nach kulturellen, sozialstrukturellen oder situativen Besonderheiten.

Eine dritte Schwerpunktsetzung der öffentlichen, wissenschaftlichen und juristischen Debatte ist die ebenfalls auf dem feministischen Erbe basierende Vorstellung, sexuelle Gewalt, darunter auch Vergewaltigungen, werde strategisch und systematisch als „Kriegswaffe“ eingesetzt (Kirby 2012, S. 806). Im Zusammenhang mit dem Krieg im ehemaligen Jugoslawien wurden Vergewaltigungen erstmals völkerrechtlich als Kriegsverbrechen und -strategie definiert und untersucht (Beck 2004, S. 60). In der jüngsten Vergangenheit wird die Kriegsstrategie-These nicht mehr unhinterfragt als Erklärungsmodell für Vergewaltigungen in kriegerischen Konflikten herangezogen. Ein Grund dafür ist ihre nicht unproblematische juristische Kopplung mit der Verurteilung von Kriegsvergewaltigung als Verbrechen gegen die Menschlichkeit. Da im juristischen Sinne erst von Kriegsverbrechen gesprochen wird, wenn eine gewisse Massenhaftigkeit und Systematik nachgewiesen werden kann (Beck 2004, S. 67), kann schon das bloße Hinterfragen der Kriegsstrategie-These als Versuch gewertet werden, die Täter*innen zu entlasten.

Diese Fokussierungen hatten zur Folge, dass bis vor einem Jahrzehnt einige Aspekte und Perspektiven, die unserer Ansicht nach für ein Verständnis bzw. eine Erklärung von sexueller Kriegsgewalt zentral sind, vernachlässigt wurden: die Dynamik der Gewaltsituation, Emotionen, langfristige soziale Habitus, militärische Strukturen, die Unterscheidung von Zivilleben und Kriegszustand, die Täterperspektive und die Unterscheidung verschiedener Arten sexueller Gewalt.

Seit einer Dekade werden viele dieser Aspekte aufgegriffen. Da bisher nur wenige soziologische Arbeiten vorliegen, müssen wir uns auf geschichtswissenschaftliche (etwa Beck 2004; Gebhardt 2015; Lilly 2007), politikwissenschaftliche (etwa Cohen und Nordås 2014; Clark 2014; Eriksson Baaz und Stern 2013; Kelly 2010; Wood 2006, 2009) und psychologische (Henry et al. 2004; Skjelsbæk 2015) Untersuchungen stützen. Viele von ihnen weisen darauf hin, dass die mannigfaltigen Formen von sexueller Kriegsgewalt nicht monokausal bzw. ausschließlich aus der Makroebene zu erklären sind. Während eine genuine Täter*innenforschung in den Geschichtswissenschaften (NS-Vergangenheit) und der Psychologie (Täterprofile) eine lange Tradition hat, wurde ihr in der Soziologie der Weg erst jüngst geebnet: z. B. durch die auf Interviews mit Bürgerkriegssoldaten aus dem Kongo basierenden Studien von Eriksson Baaz und Stern (2010) und Kelly (2010) oder durch Skjelsbæks (2015) narrative Analyse von Gerichtsurteilen zum Jugoslawienkrieg. Erwähnenswert ist auch Eriksson Baaz' und Sterns (2013, S. 79 ff.) Versuch, Collins' Vorwärtspanik-Konzept auf sexuelle Gewalt im kongolesischen Bürgerkrieg anzuwenden.

Trotzdem sind zentrale Begriffe bisher nicht geklärt. In dieser Begriffsvielfalt spiegelt sich einerseits die seit Brownmiller diskutierte Frage wider, ob eine Vergewaltigung als gewaltsame Ausübung sexueller Handlungen oder als sexuelle Ausübung von männlicher Macht (,,sexualisierte Gewalt“) zu verstehen sei. Andererseits werden die Begriffe „Kriegsvergewaltigung“ und „,sexuelle Kriegsgewalt“ sehr unterschiedlich verwendet: von einem engen Verständnis, das sich auf den Akt der 
Penetration eines menschlichen Körpers beschränkt, bis zu breiteren Auffassungen, die institutionalisierte Formen (z.B. Comfort Women, Rape Camps) oder gesellschaftliche Machtbeziehungen einschließen. Zwar wurden Versuche der begrifflichen Klärung unternommen (siehe Isikozlu 2011; Kelly 2010), jedoch steht eine systematische begriffliche Abgrenzung aus.

Um diesem Umstand Rechnung zu tragen, halten wir es für angemessen, das begriffliche Instrumentarium aus dem empirischen Material zu entwickeln. In der Zwischenzeit müssen wir uns mit unzureichenden Begriffen begnügen. Und da ist aus unserer Sicht für ein soziologisch-entdeckendes Vorhaben der Begriff ,,sexuelle Gewalt“ dem in der feministischen Tradition wurzelnden Begriff ,sexualisierte Gewalt" vorzuziehen. Während der zweite Ausdruck von vornherein nahelegt, sexuelle Gewalt sei immer und ausschließlich als Sexualisierung von Gewalt zu verstehen, erlaubt es der erste, die ganze Bandbreite der empirisch potenziell beobachtbaren Phänomene (und die Übergänge und Beziehungen zwischen ihnen) in den Blick zu bekommen. Der Begriff ,sexuelle Gewalt“ lässt die Frage zu, unter welchen Umständen Gewalt sexualisiert oder Sexualität violentisiert wird, wo die Grenzen zwischen sexueller und nicht-sexueller Gewalt, zwischen erzwungener und nicht-erzwungener Sexualität sowie zwischen Krieg und Zivilleben verlaufen und wie sich diese Grenzen verschieben.

Es ist an dieser Stelle nicht möglich, auf alle Forschungslücken - wie bspw. eine überfällige begriffliche Auseinandersetzung und eine damit verbundene Typisierung sexueller Kriegsgewalt - einzugehen. Einige von ihnen werden im Rahmen der untenstehenden Diskussion thematisiert.

\section{Krieg und (sexuelle) Gewalt bei Collins und Elias}

Im Folgenden möchten wir zwei in der soziologischen Gewaltforschung etablierte Ansätze - Collins' mikrosoziologische Gewalttheorie und Elias' figurationstheoretische Soziologie - auf ihre Eignung für die Analyse von sexueller Kriegsgewalt überprüfen. Zunächst werden wir danach fragen, was sie zum Verständnis von sexueller Kriegsgewalt beitragen können.

\section{1 (Sexuelle) Gewalt bei Collins}

Nachdem die Rezeption von Collins' früheren, makro- und konfliktsoziologischen Arbeiten vornehmlich auf den englischsprachigen Raum beschränkt war, stieß die deutsche Übersetzung seiner mikro- und gewaltsoziologischen Grundsatzschrift Violence: A Micro-Sociological Theory (2008; deutsch 2011a) auch in Europa auf breite Resonanz. Vor allem im deutschsprachigen Raum ist Collins hauptsächlich als mikrosoziologischer Gewaltforscher bekannt, während seine makrosoziologische Konfliktsoziologe - die immerhin die ersten 25 Jahre seines Schaffens prägte (Collins 2019, S. 60) - dort bis heute oft vernachlässigt wird. ${ }^{3}$

\footnotetext{
3 Ausnahmen sind z. B. Joas und Knöbl (2004) und Rössel (2012).
} 


\subsubsection{Mikro und Makro}

Collins' frühe konflikttheoretische Ausrichtung ist maßgeblich durch Marx und Weber beeinflusst. Auch die Arbeiten seines Lehrers Reinhard Bendix dürften zur Orientierung an einer makrosoziologischen Konflikttheorie und einer historisch vergleichenden Makrosoziologie beigetragen haben (Rössel 2012, S. 7 ff.). In der 1999 unter dem vielsagenden Titel Macrohistory: Essays in Sociology of the Long Run (1999) erschienenen Essaysammlung entfaltet Collins eine geopolitische Perspektive, die nicht zuletzt durch die Historische Soziologie inspiriert ist. Der Gegenstand der Analysen reicht von Staaten, Ethnien und Märkten bis zu Revolutionen, Demokratisierungsprozessen und Kapitalismus. Wenngleich Krieg und Gewalt in diesen Frühschriften kaum adressiert werden, lassen die vielfältigen Hinweise (von Goldstone über Skocpol bis zu Mann und Tilly) kaum vermuten, dass Collins heute einer makrohistorischen bzw. -soziologischen Weiterentwicklung seiner gewaltsoziologischen Konzepte ablehnend gegenüber steht. Dass er diese Weiterentwicklung (zumindest bisher) ebenso unterlassen hat wie das Setzen einer Klammer, die seine makro- und mikrosoziologischen Ausführungen zusammenhält, ist ihm mehrfach zum Vorwurf gemacht worden (siehe zuletzt einige Beiträge in Hoebel und Malthaner 2019). ${ }^{4}$

Collins' mikrosoziologische Drift ist im Zusammenhang mit der allgemeinen Abkehr von der bis dahin vorherrschenden makrosoziologischen Ausrichtung der Ursachen-orientierten Gewaltforschung zu verstehen, die im angelsächsischen Raum in den 1970er Jahren und in Deutschland in den 1990er Jahren einsetzte. Während in der soziologischen Gewaltforschung die bis dahin dominierende, nach vorausliegenden Gewaltursachen fragende (etwa „,strukturelle Gewalt“ (Galtung 1975)), „Faktoren-Soziologie“ (Sutterlüty 2015) von einer ,genuinen Gewaltforschung“ (Trotha 1997) phänomenologischen und situationistischen Zuschnitts in Frage gestellt wurde, die das Gewalthandeln und -erleiden selbst in den Fokus stellt (Sofsky, Reemtsma, Collins), ist im Hinblick auf sexuelle Kriegsgewalt keine radikale Abkehr von der Makro-Perspektive zu erkennen.

Allerdings können Studien, die Kriegsvergewaltigung im Stile der „,classic explanations of conflict-related sexual violence" (Wood 2014, S. 462) mit bestimmten sozialstrukturellen oder kulturellen Merkmalen der Konfliktparteien darzulegen suchen (seien es ,nationale Habitus“, patriarchale Gesellschaftsstrukturen (,militarized masculinity") oder ethnisch-kulturelle bzw. religiöse Unterschiede), nicht erklären, warum in verschiedenen Kriegen ähnliche Muster des Gewaltverslaufs und insbesondere der sexuellen Gewalt zu beobachten sind - unabhängig von makrosoziologischen Merkmalen der beteiligten Gesellschaften. Auch sind sie nicht geeignet zu erklären, warum innerhalb ein und desselben Krieges Art, Ausmaß und Intensität der

\footnotetext{
${ }^{4}$ Von einer Einbeziehung von Collins' makrosoziologischen Arbeiten wird hier abgesehen, da uns die Fruchtbarmachung der mikrosoziologischen Gewalttheorie angesichts des Fehlens einer Mikroperspektive auf sexuelle Kriegsgewalt dringlicher erscheint. Zudem sind Collins' neuere Aufsätze durch ihre ,polemisierende Gestik" (Collins 2019, S. 60) für die Kontrastierung mit anderen Perspektiven prädestiniert. Dass der Dialog zwischen mikro- und figurationssoziologischer Perspektive dem Erarbeiten einer sozialtheoretischen Collins-Klammer vorgezogen wird, ist der Überzeugung geschuldet, dass dieser Dialog sowohl für die Konzeption von sexueller Kriegsgewalt als auch für die beiden Perspektiven anregend sein kann.
} 
(sexuellen) Gewalt variieren - warum es also in einer Schlacht zu Massakern kommt und in der nächsten nicht; warum ein Trupp im Anschluss an eine Kriegshandlung vergewaltigt, nach einer anderen Kriegshandlung nicht; und warum der Befehl zur Tötung der wehrfähigen Männer einmal in ein rauschhaftes und unkontrollierbares Ermorden und Vergewaltigen von Frauen, Alten und Kindern ausartet, ein paar Tage zuvor jedoch nicht. Seit etwa einem Jahrzehnt werden Ansätze entwickelt, die sexuelle Kriegsgewalt aus einer Meso-Perspektive untersuchen (z. B. indem sie militärische Strukturen einbeziehen). Empirische und theoretische Arbeiten, die sexuelle Kriegsgewalt systematisch aus einer mikrosoziologischen Perspektive untersuchen, fehlen hingegen nach wie vor.

\subsubsection{Collins' mikrosoziologische Gewalttheorie}

Obwohl sich Collins' Theorie der Gewalt auf unterschiedlichste Gewaltformen bezieht, hat er sich bisher nicht explizit mit sexueller Gewalt im Krieg beschäftigt. Zwar finden sich in Violence im Zusammenhang mit dem Nanking-Massaker (1937) Anmerkungen zu Vergewaltigungen (Collins 2008, S. 97 ff., 463). Eine systematische Analyse fehlt jedoch. Dies ist vermutlich auch - wenngleich sicher nicht nur mit der schwierigen Quellenlage zu erklären, die eine materialnahe Rekonstruktion, auf der Collins' Situationsanalyse beruht, erschwert. Allerdings wäre zu fragen, was dies über die Grenzen und Potenziale einzelner theoretischer Konzepte der collinsschen Gewalttheorie (und über Collins' diesbezügliche Einschätzungen) aussagt.

Unabhängig davon bieten seine aktuelleren Arbeiten zahlreiche Anschlussmöglichkeiten für weiterführende Überlegungen. Erstens gewährt die mikrosoziologische Gewalttheorie - insbesondere die Konzepte der „Konfrontationsanspannung und -angst“, der „,emotionalen Dominanz“ und der „Vorwärtspanik“ - Ausgangspunkte zur Untersuchung bestimmter Typen der sexuellen Kriegsgewalt. Zweitens hat sich Collins ebenfalls mit Sexualität beschäftigt. Und drittens soll der seit längerem angekündigte Folgeband von Violence Ausführungen zum Thema Vergewaltigung enthalten.

Insgesamt ist Collins innerhalb der soziologischen Gewaltforschung einer der prominentesten und streitbarsten Vertreter der Mikroperspektive. Er untersucht Gewalt als soziale Interaktion. Dabei legt er den Fokus weder auf einzelne Gewalttäter*innen noch auf „Hintergrundfaktoren“ (Collins 2011a, S. 36) wie soziale Herkunft, Ethnizität, Geschlecht, Alter oder familiäre Verhältnisse, sondern auf die Gewaltsituation selbst. Ob und wie Gewalt ausgeübt wird, bestimmen die situativen Dynamiken und Emotionen vor Ort.

Im Zentrum steht das Konzept der „Konfrontationsanspannung und -angst“ (Collins 2011a, S. 19). Collins' These ist, dass die Ausübung von Gewalt niemals leichtfällt. Gelinge es nicht, dem Konflikt auszuweichen - was im Krieg besonders häufig der Fall ist -, stellten sich Anspannung und Angst ein. Daher biete das Kriegsgeschehen ideale Voraussetzungen für den Aufbau und die Verlängerung von Anspannung. Zentral für erfolgreiche Gewalt sei, dass Konfrontationsanspannung und -angst entweder vermieden oder umgangen wird - bei organisierten publikumsorientierten Duellen (,fair fights“), durch große Entfernung vom Feind (Distanzwaffen), mittels Überraschungsangriffen, durch spezielles Training oder durch Angriffe auf 
einen eindeutig schwächeren Gegner. Der letztgenannte Aspekt ist beim Konzept der „Vorwärtspanik“ zentral, das für die Untersuchung von sexueller Kriegsgewalt im Zusammenhang von Massakern besonders vielversprechend ist (Collins 2011b, S. 157). Zeige nämlich nach einer längeren, schwierigen Konfrontation eine Seite eine Schwäche, stelle sich bei der anderen Seite ein Überlegenheitsgefühl ein, und die Anspannung entlade sich schlagartig in einer panischen Flucht nach vorne. In diesem Zustand der kollektiven Raserei werde Gewalt länger und massiver ausgeübt, als für den Sieg notwendig sei (,Overkill“); es komme zu Kriegsgräueln (Collins 2015, S. $204 \mathrm{ff}$.).

Allerdings geht Collins (2011a, S. 153f.) nicht davon aus, dass alle Kriegsgräuel Folge einer Vorwärtspanik sind. Er nennt folgende Einschränkungen: den militärischen oder politischen Befehl (z. B. ,,rassische“ Säuberungsaktionen des NSRegimes an der Ostfront); eine Politik der verbrannten Erde, um den Kriegsgegner seiner Ressourcen zu berauben (wie im Ostfeldzug der Wehrmacht) und den Kampf gegen Widerstandsbewegungen (z.B. Partisanenbekämpfung). Das potenzielle Ursachenbündel beinhaltet somit auch rationale Momente und strategische Entscheidungen, womit Kriegsstrategien als eine mögliche - aber nicht die einzige - Ursache für Kriegsgräuel einbezogen werden.

Für die Untersuchung von sexueller Kriegsgewalt bietet Collins' mikrosoziologische Gewalttheorie einige Anknüpfungspunkte. Erstens ermöglicht der emotionssoziologische Ansatz (v.a. die Konzepte „Emotionale Energie“ und „Vorwärtspanik“) eine kritische Auseinandersetzung mit der Kriegsstrategie-These. Während der Begriff „Strategie“ nicht nur ein Bild vom rational handelnden Soldaten, sondern auch vom Kriegsschauplatz als emotionsfreiem Raum impliziert, legt Collins den Fokus auf emotionale Spannungen und Interaktionsdynamiken in der Konfrontationssituation. Zweitens bieten die detaillierte Rekonstruktion und die emotionssoziologische Analyse von Gewaltsituationen (und deren Genese) die Möglichkeit, Voraussetzungen und Dynamiken verschiedener Arten von sexueller Kriegsgewalt auszuarbeiten. Drittens sind die von Collins (2009) eingeführten, aber noch nicht systematisch ausgeführten Konzepte der „Moralischen Auszeit““ und der „Publikumsorientierten Massengewalt" von Interesse.

\section{2 (Sexuelle) Gewalt bei Elias}

Im Gegensatz zu Collins wird Elias kaum als Gewaltsoziologe im engeren Sinne rezipiert. Nicht ganz zu Unrecht, schließlich weicht sein - ebenfalls an Marx und Weber orientiertes - Grundproblem, eine Zentraltheorie der Menschheitsentwicklung zu entwerfen, die als Bezugsrahmen für alle Gesellschaftswissenschaften dienen kann, recht deutlich von der Vorstellung einer spezialisierten (,genuinen“) Gewaltforschung ab.

\subsubsection{Gewalt und Zivilisierung auf der innerstaatlichen Ebene}

Trotzdem nimmt die Frage, warum Menschen Gewalt ausüben und Krieg führen, in seinem Werk eine bedeutende Rolle ein. Welchen zentralen Stellenwert Elias gewalttätigen Konflikten und körperlicher Gewalttätigkeit im Zusammenleben der 
Menschen einräumt, zeigt seine Forderung, die Fragestellung der Gewaltforschung umzudrehen. Das Rätsel sei nicht, warum Menschen einander schlagen oder erschlagen, sondern wie es möglich ist, dass so viele Menschen friedlich miteinander leben können, ohne ständig fürchten zu müssen, von Stärkeren geschlagen oder erschlagen zu werden. Schließlich hätten noch nie in der Menschheitsgeschichte so viele Menschen so friedlich neben- und miteinander gelebt wie in den großen Staaten der Gegenwart (siehe Elias 2006a, S. 74).

Elias erklärt Gewalt(-bereitschaft) nicht als Resultat situativer Dynamiken, sondern u.a. als Teil eines psychischen „Habitus“, der das Ergebnis gesellschaftlicher „Prägeapparaturen“ und Prozesse sei. Weil Konflikte ein normaler Aspekt des Zusammenlebens seien, könne man sie nicht vom isolierten Individuum her erklären, sondern nur aus der Art, wie Menschen in Gruppen miteinander verbunden sind (Elias 2006a, S. 72 ff.).

Elias' Soziologie liegt die in Auseinandersetzung mit Marx und Mannheim entwickelte Idee zugrunde, dass sich im Zuge gesellschaftlicher Wandlungsprozesse die affektiven Strukturen, Verhaltensmuster und Geschmäcker jener Menschen wandeln, die diese sozialen „Figurationen“ (Elias 2006d, S. 100 ff.) miteinander bilden. Triebkraft dieses Wandels sei der ständige Konkurrenzkampf, durch den Menschen und Gruppen aneinander gebunden sind. Obwohl Elias nicht davon ausgeht, dass sich solche Integrations- und Desintegrationsprozesse linear und überall gleichförmig vollziehen, verlaufe der gesellschaftliche Wandel nicht strukturlos, sondern lasse sich in vielen Gesellschaften auf lange Sicht als Entwicklung in eine bestimmte Richtung beschreiben (Elias 2006c, S. 389 ff.; 2006e, S. 182 ff.; 2006f): in Richtung „Zivilisierung“. 5

Im Hinblick auf die in kriegerischen Konflikten ausgeübte und erlittene sexuelle Gewalt spielt die in den kriegführenden Staaten vorherrschende Machtbalance zwischen den Geschlechtern (Elias 2006b) eine Rolle, die über Geschlechterbilder, Sexual- und Gewaltnormen von Befehlshaber*innen, Soldat*innen und potenziellen Opfern in die Gewaltsituation einwirken. ${ }^{6}$ Da gesellschaftliche „Prägeapparaturen“ (Schule, Kirche, Militär, Bürokratie) verschiedene soziale Schichten unterschied-

\footnotetext{
5 In seinen Prozess-Büchern zeichnet Elias (1936/1939, hier 1997) diesen Prozess beispielhaft für das europäische „Abendland“ vom Mittelalter bis zum Ersten Weltkrieg nach. Die Entstehung immer gröBerer pazifizierter Räume und immer stabilerer Gewaltmonopole habe gemeinsam mit der Ausdehnung wirtschaftlicher Abhängigkeitsnetze eine Verfeinerung der Sitten erzwungen - zunächst durch immer stärker werdende Fremdzwänge (soziale Sanktionen), später durch immer stärker internalisierte Selbstzwänge (Voraussicht, Schamgefühle, Ängste). So habe sich über die Jahrhunderte ein sozialer Habitus entwickelt, der sich sowohl durch eine zunehmende Kontrolle, Verbannung und Umlenkung von Aggressivität und Gewalttätigkeit als auch durch eine wachsende Kontrolle, Unterdrückung und Tabuisierung von Nacktheit und Sexualität auszeichnet. Ein idealtypischer Zivilisationsprozess ist gekennzeichnet durch eine abnehmende Ungleichheit zwischen den Geschlechtern (Elias 2006b), eine De-Violentisierung von Sexualität und eine Ent-Sexualisierung von Gewalt. Die These des historischen Gewaltrückgangs findet sich aktuell etwa bei Pinker (2011) oder Linklater (2017); kritisch siehe mehrere Beiträge in Sutterlüty et al. (2019).

6 Unlängst wurden verschiedene Aspekte des Geschlechterverhältnisses aus zivilisationstheoretischer Perspektive untersucht: der Wandel weiblicher Körperbilder, Verhaltensnormen und Anstandsregeln (Wouters 2004); die Erotisierung und Tabuisierung von Nacktheit (Górnicka 2016); weibliche Teilhabeaspirationen, männliche Abwehr- und Rückzugsreaktionen sowie Selbst- und Fremdsexualisierung (Ebner 2018, S. $241 \mathrm{ff}$.).
} 
lich prägen, können sich innerhalb eines Staates unterschiedliche Variationen des nationalen Habitus ausbilden. ${ }^{7}$

Für die Untersuchung von sexueller Gewalt im Krieg wäre demnach zu fragen, ob und wie sich die Struktur der Beziehungen innerhalb der Kriegsparteien darauf auswirkt, ob, wie und warum unter welchen situativen Umständen welche Art der (nicht-sexuellen und sexuellen) Gewalt bzw. der (nicht-gewaltvollen und gewaltvollen) Sexualität zu beobachten ist (Elias und Scotson 1993). Folglich wären innerstaatliche Konkurrenzdynamiken und Machtgefälle, die Teilhabemöglichkeiten verschiedener Gruppen, deren jeweils erwartete Machtgewinne bzw. -verluste durch einen Kriegsgewinn oder die jeweiligen sinnstiftenden Funktionen des Krieges einzubeziehen. Auch sozio- und psychogenetische Charakteristika der militärischen Apparate könnten betrachtet werden.

\subsubsection{Gewalt als Struktureigentümlichkeit zwischenstaatlicher Ausscheidungskämpfe}

Aus dieser Perspektive wäre auch zu fragen, wie die (sexuelle) Gewaltbereitschaft im Krieg mit der Struktur der Staatenfiguration und der jeweiligen Position der kriegführenden Staaten in diesem Konkurrenzgeflecht zusammenhängt. ${ }^{8}$ Die Beziehungsdynamik zwischen Kriegsparteien könnte z.B. beeinflussen, ob, warum und wie es im Laufe eines Konfliktes zu sexueller Gewalt kommt bzw. ob nicht-sexuelle in sexuelle Gewaltformen und nicht-gewaltvolle in gewaltvolle Sexualnormen umschlagen. Einerseits ist denkbar, dass dabei strategische Überlegungen (Demütigung als psychologische Kriegswaffe, Auslöschen des Feindes) eine Rolle spielen. Insbesondere ist aber davon auszugehen, dass der sich im Zuge vorangegangener Konflikte entsponnene Kreislauf gegenseitiger Bedrohungen und Beschämungen in den Persönlichkeitsstrukturen der Akteure Spuren hinterlässt (Elias 2006a, S. 81 ff.), die sich auf deren Gewaltbereitschaft auswirken. Man denke an Ängste, Demütigungen, Scham-Wut-Spiralen (Scheff 1994; Scheff und Retzinger 1997), Hassund Rachegefühle, enttäuschte Hoffnungen, Feindbilder, Überlegenheitsgefühle, die Kompensation von Minderwertigkeitsgefühlen oder militärischen Niederlagen.

\footnotetext{
${ }^{7}$ Der Habitus der Eliten kann ,zivilisierter“ sein als jener der breiteren Bevölkerungsschichten (und als Distinktionsmittel nach unten dienen), er kann aber auch hinter ihnen zurückhinken (z. B., wenn sich Eliten an einem älteren Ethos orientieren).

${ }^{8}$ Für Elias (2003c, S. 219 ff.) liegt die Ursache für Krieg weder in den rationalen Plänen einzelner Staatsoberhäupter noch in der besonderen Aggressivität eines „Volkes“ oder einer angeborenen männlichen Angriffslust begründet, sondern im immanenten Konkurrenzdruck, durch den soziale „Überlebenseinheiten“ (seien es Stämme, Schichten, Fürstentümer, Staaten oder ethnisch definierte Gruppen) gegeneinander getrieben werden. Im Gegensatz zur innerstaatlichen Ebene konnte sich in den zwischenstaatlichen Beziehungen bis heute kein wirksames Gewaltmonopol etablieren. Daher beruhe das relative Machtpotenzial eines Staates noch immer stärker auf der Fähigkeit und Bereitschaft zur Anwendung physischer Gewalt. Diese Figurationsdynamik binde Staaten so aneinander, dass jeder eine potenzielle Gefahr für den anderen darstellt und keiner diese Gefahr ausschalten oder kontrollieren kann. Dem hohen Gefahrenniveau entsprechend, zeichneten sich zwischenstaatliche Beziehungen durch eine vergleichsweise hohe Affektgeladenheit des Denkens, eine geringe Fähigkeit zur emotionalen Selbstkontrolle und folglich eine Unfähigkeit der Beendigung dieses Kreislaufes aus.
} 
Ein weiterer Aspekt ist, dass sich, den unterschiedlichen Beziehungsdynamiken auf inner- und zwischenstaatlicher Ebene entsprechend, die Standards des zivilisierten Verhaltens voneinander unterscheiden können: Dem in den meisten industrialisierten Nationalstaaten vorherrschenden Kanon der Gewaltlosigkeit steht in den zwischenstaatlichen Beziehungen (nicht nur im Kriegsfall) ein Kanon gegenüber, der physische Gewalt fordert und belohnt (Elias 2003c, S. $228 \mathrm{ff}$.). Gerade mit Blick auf sexuelle Gewalt könnte es sich lohnen zu fragen, inwiefern zwischenstaatlichmilitärische von innerstaatlich-zivilen Verhaltenskanons abweichen und die persönlichen Spannungen und Kompensationsstrategien von Berufssoldat*innen und Wehrpflichtigen in den Blick zu nehmen. Zudem ist der im Kriegsfall geforderte Kanon nicht allen Mitgliedern pazifizierter Nationalstaaten gleichermaßen fremd: während für das Gros der Bevölkerung ein Kanon totaler Gewaltlosigkeit gilt, ist etwa die Polizei auch in Friedenszeiten zur Gewaltausübung autorisiert (ähnlich Collins zu „Gewaltspezialisten“").

\subsubsection{Zivilisierung des Krieges und Entzivilisierung im Kriegsgeschehen}

Der letzte Anknüpfungspunkt, auf den wir hier hinweisen wollen, ist die Erfahrung des Krieges. Dem Ideal eines Hin und Her zwischen Mikro- und Makroperspektive folgend, hat Elias Gewaltsituationen aus der Perspektive der Täter analysiert (z.B. den Furor des mittelalterlichen Kriegers beim Plündern, Schänden und Verstümmeln wehrloser Bauernfamilien (1997); oder die Brutalität der Freikorps zur Zeit der Weimarer Republik (2006a, S. 90 ff.)). Zwar räumt er situativen Auslösern (enttäuschte Siegeshoffnungen, unerwarteter Widerstand, Gefühl des Verrats) einen Stellenwert ein, als entscheidend erachtet er aber Ereignisse und Strukturen, die der unmittelbaren Gewaltsituation vorausgehen (nationales Unterlegenheitsgefühl, Umschlag in Überlegenheitsgefühl durch militärische Siege, Ausbreitung eines kriegsbejahenden Ehrenkanons, sinnstiftende Funktion des Krieges, mangelnde Identifizierung mit dem Gegner, persönliche Hoffnungen auf einen Sieg oder enttäuschte Hoffnungen durch Niederlagen).

Ein prominentes Beispiel für die Frage, wie längerfristige Entwicklungen sich darauf auswirken, welche Gewaltsituationen entstehen, wie sie sich gestalten, welche „situativen Zwänge“ sich daraus ergeben und wie sie erlebt werden, ist jene nach der „Zivilisierung des Krieges“. Mehr als Elias selbst haben an ihn anknüpfende Soziolog*innen auf den Widerspruch zwischen der Zivilisierung des Ausübens und der Entzivilisierung des Erleidens von Gewalt hingewiesen. Einerseits ermöglichen moderne, ferngesteuerte Waffensysteme eine Vergrößerung der räumlichen und emotionalen Distanz zwischen den Kombattanten, wodurch es seltener zu Nahkampfsituationen komme und - zumindest aufseiten der Gewaltausübenden - weniger Hass, Angriffslust und Todesangst zu erwarten sei. Andererseits resultiere die gesteigerte Effizienz neuerer Waffensysteme in einer geringeren Identifikation mit dem Feind sowie in Wut- und Rachegefühlen über die verheerenden Ausmaße; zudem werde die Unterscheidung zwischen Krieger*innen und Zivilbevölkerung schwieriger (Kuzmics 2015, S. 210 ff.; ähnlich Collins 2009, S. 17 ff.).

Besonders relevant für sexuelle Kriegsgewalt erscheint die „Entzivilisierungs“These, die das Senken gesellschaftlich erlernter Scham- und Peinlichkeitsschwellen 
hinsichtlich Angriffslust, Sexualität, Angst und Mitgefühl adressiert. Während Elias (2006a, S. 104f.) davon ausgeht, dass der Auflösung zivilisierter Verhaltens- und Gewissensformen fast immer ein langer gesellschaftlicher Prozess der Gewissenszersetzung vorausgeht, fragen rezente empirische Arbeiten aus einer stärker mikrosoziologischen Perspektive nach der entzivilisierenden Wirkung des Kriegsgeschehens. ${ }^{9}$

\section{Gemeinsamkeiten und Unterschiede zwischen Collins und Elias}

Nachdem nun die Prämissen der beiden Ansätze skizziert sind, werden diese zunächst miteinander kontrastiert und danach kritisch betrachtet. Vorab lässt sich festhalten, dass es trotz aller Unterschiede erstaunliche Gemeinsamkeiten gibt. Wie wir zeigen werden, sind auch die Differenzen fruchtbar, um auf die jeweiligen Defizite aufmerksam zu machen. ${ }^{10}$

Da die Überlappungen unten ausführlich diskutiert werden, werden sie hier nur kurz angeführt. Die augenscheinlichste Gemeinsamkeit ist, dass Collins und Elias Gewalt(-bereitschaft) empirisch zu studieren und Wertungen und „Mythen“ zu überwinden trachten. Dies gilt sowohl für pessimistische und verurteilende Einschätzungen als auch für popkulturell geprägte überhöhende bzw. beschönigende Darstellungen. Zweitens ist ihnen eine relationale und prozessuale Argumentationsweise gemein. Die dritte Ähnlichkeit ist, dass physische Gewalt in modernen Gesellschaften außerhalb von Enklaven (im Krieg, beim Sport, unter Kindern) als Ausnahmefall betrachtet wird. Viertens konzipieren Collins und Elias Gewaltbereitschaft weder als angeboren noch als von einem Pol her erklärbar. Fünftens räumen sie Emotionen einen hohen Stellenwert bei der Ausübung von physischer Gewalt ein. Beide nehmen an, dass physischer Gewalt starke, nicht rational geprüfte Emotionen vorausgehen - was angesichts Collins' symbolisch-interaktionistischer Prägung durchaus bemerkenswert ist (Kuzmics 2015).

Der offenkundigste Unterschied betrifft das Verhältnis von Individuum und Gesellschaft bzw. Mikro- und Makro-Ebene. Collins' Vorgehensweise zeichnet sich dadurch aus, dass einzelne Situationen weitgehend isoliert voneinander untersucht werden. Zwar ist er einer Makroanalyse von Gewalt gegenüber nicht abgeneigt (Collins 2009), jedoch müsse ihr immer eine Mikroanalyse vorausgehen (Kuzmics 2015, S. 221 f.; Kuzmics und Haring 2013, S. 527). Im Zusammenhang mit „Makrogewalt" (sei es in der Form von Kriegen oder Auseinandersetzungen zwischen paramilitärischen Gruppen) betont er die Bedeutung großer Organisationen (Staaten,

\footnotetext{
9 Mit Blick auf sexuelle Gewalt ist insbesondere de Swaans Monographie The Killing Compartments (2015) instruktiv. Kuzmics und Haring (2013) untersuchen hingegen anhand der Kriegstagebücher von Soldaten und Offizieren der Habsburger Armee das psychische Erleben des Kriegsalltags. Der von ihnen identifizierte „Verlust der Moral“ ist vergleichbar mit Collins’ „Moral Holidays“, wenngleich sie neben situativen Dynamiken des Frontgeschehens (Widerstand, Dauerbeschuss, Niederlagen, Spionageangst, Solidarität) auch längerfristige Makroprozesse (Konkurrenzdynamik zwischen den Dynastien, Entfremdung zwischen deren Eliten, Mentalitätsunterschiede und Nationalismen innerhalb der k.u.k. Armee, Offiziershabitus, Versorgungsengpässe, Stolz auf vorangegangene Schlachten) einbeziehen.

10 Die Ausführungen dieses Abschnittes basieren auf zwei bilanzierenden Aufsätzen von Kuzmics (2015; Kuzmics und Haring 2013, S. 493 ff.).
} 
Armeen, soziale Bewegungen). Sie hätten die Aufgabe, mittels materieller, sozialer und emotionaler Ressourcen ,erfolgreiche Gewalt“ zu ermöglichen und aufrechtzuerhalten - das heißt: das Entstehen von Konfrontationsanspannung und -angst zu verhindern. Konkret bedeutet das: Trainieren, Versorgen, Transportieren, Disziplinieren, von der Flucht Abhalten (Collins 2009, S. 17 ff.). ${ }^{11}$ Elias' (2001) Soziologie zeichnet sich hingegen durch den Anspruch aus, philosophisch-idealistische und soziologische Dualismen ${ }^{12}$ zu überwinden. Sein Fokus liegt nicht auf einzelnen Situationen, sondern auf Figurationen wechselseitig voneinander abhängiger Individuen und Gruppen (Elias 2006d, S. $100 \mathrm{ff}$.). Da einzelne Situationen erst durch den gesamten Gesellschaftsaufbau und die Struktur der Beziehungen zwischen ihnen (also ihre Vorgeschichte) ihre Relevanz und Bedeutung erhalten, ist die isolierte Untersuchung einzelner Situationen wenig erfolgversprechend.

Die zweite Differenz betrifft den Stellenwert sozialer Prozesse. Collins (2007, S. $431 \mathrm{ff}$.) ist gegen eine Herleitung gegenwärtiger Persönlichkeitstypen aus der Vorgeschichte. Im Zentrum von Elias' prozesssoziologischer Perspektive steht hingegen die Vorstellung, dass den Veränderungen der Beziehungsstrukturen bestimmte Veränderungen der psychischen Strukturen der Menschen entsprechen. Folglich leitet er aktuelle Persönlichkeitstypen aus der Vorgeschichte ab. Da Elias den Habitus als einen nie abgeschlossenen Prozess versteht, kann sich in einem „,nationalen Habitus“ die wechselvolle politische Geschichte einer Staatsgesellschaft widerspiegeln (Elias 2006a, S. 81 f.). Auf diese Weise kann der persönliche Habitus eines Menschen, der ebenfalls nie abgeschlossen ist, auch von früheren Phasen der Staatsgenese geprägt sein (Kuzmics 2015, S. 227 f.; Kuzmics und Haring 2013, S. 527).

Auch bezüglich des Stellenwerts der Zeit-Dimension gibt es Unterschiede. Da sich Collins' Methode durch die Rekonstruktion und die situationsbezogene Erklärung von Gewaltdynamiken auszeichnet, untersucht er ebenfalls Prozesse. Der zentrale Unterschied zu Elias besteht darin, dass Collins' Zeithorizont wesentlich kürzer ist. Allerdings sind viele seiner empirischen Analysen durch ein recht großzügiges Situationsverständnis gekennzeichnet; etwa wenn er Gewalthandlungen über Monate hinweg als eine „Situation“ untersucht, oder wenn er Ereignisse, die lange vor der untersuchten Gewaltsituation stattgefunden haben, in die Situationsanalyse einbezieht (Collins 2011a, S. 154 f.). Collins fordert selbst ein ,model incorporating time-dynamics“ (Collins 2009, S. 20), das erklärt, wie lange soziale und emotionale Ressourcen (z. B. Gruppensolidarität nach einem Angriff, oder Eskalation und Deeskalation) wirksam sind. ${ }^{13}$ Elias' Analysen sind hingegen historisch und raum-zeitgebunden. Er erklärt zeitgenössische Affektmodellierungen als Resultat langfristiger sozialer Prozesse (Kuzmics 2015, S. 226). Der zentrale Unterschied zu Collins besteht darin, dass bei Elias psychische Prägungen auch dann wirksam bleiben können,

\footnotetext{
11 Möglicherweise enthält das mit Spannung erwartete Buch Theory of Emergent Conflict Analysen von „Makro-Konflikten“, in denen auch situative und zeitliche Dynamiken untersucht werden (Collins 2019, S. 60).

12 Mikro-Makro, Individuum-Gesellschaft, Handlung-Struktur, Geist-Körper, Kognition-Emotion, NaturKultur.

13 Bei Aufständen geht er von wenigen Tagen aus, bei zwischenstaatlichen Kriegen von einigen Jahren und bei Guerilla-Kriegen von mehreren Jahrzehnten.
} 
wenn ein Konflikt längst beendet ist und die auf ihn bezogenen Emotionen lange abgeflaut oder vergessen sind.

Als vierter Unterschied ist der Generalisierungsanspruch anzuführen. Collins' Ambition ist, jede Art von Gewalt zu erklären - in Gegenwart oder Vergangenheit. Elias' Theoriebildung ist ebenfalls generalisierend - etwa im Hinblick auf bestimmte Prozess- und Verflechtungsmuster (,Richtungsbeständigkeit“, „Konkurrenzmechanismus"). Aber er bestreitet, dass sich für den Gegenstandsbereich der Soziologie zeit- und ortsunabhängige Gesetze aufstellen lassen - womit er sich vom philosophischen a-priori-Denken ebenso abgrenzt wie von Kausalmodellen der quantitativen Sozialforschung (Elias 2003a, S. 136 ff.). Zwar ließen sich auch in sozialen Figurationsprozessen allgemeine Regelmäßigkeiten beobachten, aber diese seien aufgrund der Reflexivität und der wechselseitigen Abhängigkeit interdependenter Menschen weder kontextunabhängig noch kausal zu erklären. In verschiedenen Figurationen (z.B. in Deutschland und Österreich) mögen ähnliche Konkurrenz- und Integrationsmechanismen am Werk und entsprechend analoge Entwicklungstendenzen zu beobachten sein. Trotzdem vollzögen sich in unterschiedlichen Nationalstaaten je verschiedene (wenngleich erklärbar verschiedene) Prozessverläufe, die sich wiederum in spezifischen - in diesem Falle nationalen - Mentalitäts- oder „Habitus““Unterschieden niederschlügen, und gleichzeitig durch sie reproduziert würden (Elias 1997).

Nicht zuletzt unterscheiden sich Collins' und Elias' Vorgehensweise dadurch, welchen Stellenwert sie dem empirischen Material zuerkennen bzw. welche Anforderungen sie an die verwendeten Quellen stellen. Collins' beobachtungsbezogene Methode basiert auf der genauen Rekonstruktion von Gewaltdynamiken (inkl. Mimik und Körpersprache), die erst durch neuere technische Entwicklungen wie die Videoanalyse möglich geworden ist. Da wir auf Quellen, die eine solche Analyse erlauben, erst seit wenigen Jahrzehnten zurückgreifen können, ist eine detaillierte Rekonstruktion situativer Dynamiken für weiter zurückliegende Ereignisse nicht in dieser Form möglich. Selbst wenn man, wie Elias, zeitgenössische Benimmbücher, Belletristik und Ratgeberliteratur aus verschiedenen Jahrhunderten heranzieht, sind Theorien über langfristige Prozesse nie im so strengen Sinne verifizierbar oder falsifizierbar wie Theorien über aktuelle oder kurzfristigere Prozesse (Kuzmics 2015, S. 222).

\section{Collins und Elias im pragmatischen Dialog ${ }^{14}$}

Auf den bisher herausgearbeiteten Gemeinsamkeiten und Unterschieden aufbauend, versuchen wir im letzten Schritt einen Dialog zwischen einer mikro- und einer figurationssoziologischen Perspektive auf sexuelle Gewalt im Krieg. Dabei gehen wir der Frage nach, ob die beiden Ansätze als Korrektiv füreinander fungieren können, um die jeweiligen Schwächen und Einseitigkeiten auszugleichen. Wir werden argumentieren, dass ein Dialog nur möglich ist, wenn man beide Ansätze nicht dogma-

14 Diese Formulierung haben wir einem bisher unveröffentlichten Manuskript von Hüttermann (2012) entlehnt, in dem Figurationssoziologie und Sozialphänomenologie zueinander in Beziehung gesetzt werden. 
tisch versteht. Daher werden wir zudem fragen, welche Ansprüche beide Positionen zumindest vorübergehend aufgeben müssten. ${ }^{15}$

\subsection{Elias als Korrektiv für Collins}

Legt man Elias' Figurationssoziologie als Korrektiv an Collins' Gewalttheorie an, ergeben sich Anregungen, die sich zu drei Aspekten zusammenfassen lassen: die räumlich-zeitliche Einbeziehung langfristiger Figurationsprozesse, mittelfristiger Dynamiken und kurzfristiger Prägungen.

\subsubsection{Raum-zeitliche Einbettung von Situationen bzw. Einbeziehung langfristiger Prägungen}

Aus unserer Sicht würde der collinssche Ansatz profitieren, wenn er, statt einzelne Situationen getrennt voneinander zu untersuchen, auch deren jeweilige Vorgeschichte (die ja ebenfalls aus Situationen besteht) einbezöge (Cooney 2009). Bei Collins kann sich eine Situation über Tage, Wochen und sogar Monate hinziehen. Aus einer prozesssoziologischen Perspektive wäre ebenfalls der raum-zeitliche Kontext dieser langen „Situationen“ einzubeziehen, der sich nicht nur in der inneren Struktur der sich bekämpfenden Groß- und Kleingruppen (Staaten, Kampfeinheiten) und in der Struktur der Beziehungen zwischen den sich bekämpfenden Gruppen (Elias 2003b, S. $285 \mathrm{ff}$.) widerspiegelt, sondern auch in den psychischen Habitus der beteiligten Individuen (z. B. sozialstrukturelle, kulturelle oder religiöse Merkmale). Diese Figurationen und Habitus wirken sich wiederum darauf aus, welche situativen Konstellationen und Dynamiken entstehen, und wie sich in deren Zuge die Individuen und Gruppen zueinander verhalten.

Für sexuelle Kriegsgewalt wären demnach sozio- und psychogenetische Besonderheiten sowohl der miteinander kriegführenden Staaten (Geschlechterverhältnisse, Stabilität des Gewaltmonopols, Sexual- und Gewaltnormen) als auch der jeweiligen militärischen Apparate (Befehlsstrukturen, Hierarchien (vgl. Sutterlüty 2017), Gewalt- und Männlichkeitsideale) einzubeziehen. Z. B. wäre das Konzept der Vorwärtspanik zu historisieren und als Ergebnis eines - individuellen wie gesellschaftlichen - Zivilisationsprozesses zu verstehen, der in verschiedenen gesellschaftlichen Großgruppen (Staaten, Schichten, Institutionen) unterschiedlich verlaufen kann (Elias 1992). Collins' These, die ,Tötungshemmung“ von Soldaten könne - unabhängig von deren Sozialisation und anderen ,Hintergrundfaktoren“- nur im Zuge bestimmter situativer Dynamiken (wie „Vorwärtspanik“ oder „Moral Holidays“) überwunden werden, impliziert die Existenz eines zivilen und/oder militärischen Wertekanons, der exzessives Töten und lustvolle (sexuelle) Gewaltanwendung jenseits dieser Situationen negativ sanktioniert. Wie Elias (1997) am Beispiel des mittelalterlichen Kriegerhabitus gezeigt hat, ist dies jedoch keineswegs in allen Gesellschaften zu jeder Zeit der Fall. Er geht sogar davon aus, dass derart pazifizierte Gesellschaf-

\footnotetext{
15 Für eine Diskussion aktueller Weiterentwicklungen und Anwendungen beider Ansätze siehe Hütter-
} mann und Ebner (2020). 
ten, die ihren Mitgliedern einen entsprechend friedlichen Habitus erlauben (und aufzwingen), in der Menschheitsgeschichte die Ausnahme sind.

Während die Tötungshemmung bei Collins als eine Art nicht weiter differenzierte anthropologische Konstante erscheint, lenkt Elias' Vorstellung eines ,zivilisierten“ Habitus als Ergebnis eines gesellschaftlichen Lernprozesses die Aufmerksamkeit auf das Verhältnis von zivilem und kriegerischem Affekthaushalt und insbesondere auf den Prozess des Übergangs vom einen zum anderen. Folglich wäre nicht davon auszugehen, dass das Töten allen Menschen zu allen Zeiten in allen Gesellschaften gleich schwerfällt. Vielmehr würde man Konfrontationsanspannung als Teil eines zivilisierten Habitus und damit als Ergebnis eines habitusbildenden Zivilisationsprozesses verstehen. Ob in einer Situation Konfrontationsanspannung entsteht und wie man damit umgeht, hängt aus dieser Perspektive damit zusammen, wie oft man zuvor schon mit ähnlichen (Gewalt-)Situationen konfrontiert war - was wiederum vom kulturellen oder historischen Kontext abhängt (z.B. ob man in Deutschland lebt und aufgewachsen ist oder in Syrien). Es wäre zu prüfen, ob und wie die Vorstellung, dass Erlebnisse und Prozesse auf das Handeln und Empfinden einwirken können, die man nicht durch eigene Wahrnehmung, Interaktionen und Interpretation selbst erworben, sondern über Sozialisationsinstanzen internalisiert hat, mit Collins' Gewalttheorie vereinbar ist.

\subsubsection{Einbeziehung mittelfristiger Figurationsdynamiken}

Aber auch ohne die Perspektive für langfristige, Jahrzehnte und Jahrhunderte dauernde, Prozesse und kollektive Prägungen zu öffnen, kann Elias' Prozessperspektive dabei helfen, einige Aspekte in den Blick zu nehmen, die man aus situationistischer Perspektive vielleicht übersehen würde. Versteht man Habitus nicht als gemeinsame Merkmale der Mitglieder einer Gruppe, die das Resultat generationenübergreifender Prägungen sind, sondern als Resultat persönlicher Erfahrungen in vorangegangenen Situationen, kann man die Situationsanalyse durchaus durch das Habitus-Konzept - und dessen vielfältige emotionale, kognitive, körperliche und distinktive Implikationen - anreichern. Generell erlaubt das Habitus-Konzept zu berücksichtigen, dass vorhergehende Erlebnisse bzw. Situationen das darauffolgende Erleben, Empfinden, Denken und Handeln beeinflussen.

Dies gilt zunächst für die Figurationsdynamik, in der die miteinander Krieg führenden Großgruppen aneinander gebunden sind. Dazu zählt nicht nur die Beziehungsdynamik zwischen den Kriegsparteien, sondern auch die Struktur der Beziehungen innerhalb der Kriegsparteien. Zudem wäre jeweils zu fragen, wie sich die damit einhergehenden Grenzziehungsprozesse, Wir-Gefühle und emotionalen Dynamiken auf die langfristigen habituellen Prägungen des Selbstzwanges auswirken - insbesondere im Hinblick auf sexuelle Bedürfnisse, ethnische Überlegenheitsgefühle und die Identifikation mit dem Feind. Die Herausforderung bestünde dabei darin, jeweils empirisch nachzuweisen, ob und wie sich der Kampfsituation vorausgehende Prägungen in der jeweiligen Kampfsituation auswirken, was etwa mithilfe 
vergleichender Untersuchungen auch für weiter zurückliegende Ereignisse möglich wäre. ${ }^{16}$

\subsubsection{Berücksichtigung kurzfristiger Prägungen}

Selbst wenn man sich ausschließlich auf vorhergehende Erlebnisse im Rahmen einer eng gefassten Kampfsituation beschränkte, wäre mit Elias zu fragen, ob tatsächlich vornehmlich die emotionale Dynamik der Situation von Belang ist oder ob nicht zudem vorangegangene Erlebnisse das darauffolgende Erleben, Empfinden, Denken und Handeln beeinflussen - auch außerhalb der Ausnahmesituation. Denn wenn man davon ausgeht, man könne einzelne Situationen getrennt von vorangegangenen Situationen untersuchen und verstehen, müsste man nicht nur unterstellen, dass alles, was Menschen in eine Situation mitbringen, für ihr Handeln irrelevant ist (Nungesser 2014, S. 792), man müsste auch annehmen, dass „Situationsfaktoren" von sich aus bestimmte Eigenschaften und Bedeutungen besitzen, die nichts mit ihrer Entstehung zu tun haben. Aus einer radikal situationistischen Perspektive haben der kriegerischen Konfrontationssituation vorausgehende Prägungen keinen wesentlichen Einfluss darauf, wie einzelne Menschen mit der emotionalen Anspannungssituation umgehen (d.h. ob sie kämpfen, wie sie kämpfen, und wie es ihnen dabei geht). In Collins' Ausführungen über sog. „kompetente Gewalttätige“, die durch Training und Drill das Töten lernen (Collins 2011a, S. 565 ff.), lässt sich die Idee transsituativer Prägungen hingegen durchaus finden - wenn auch als Ausnahme von der Regel.

Mit Elias (1992, 2006e) wären Habitus-Veränderungen im Sinne einer „Entzivilisierung“ im Laufe des Kriegsgeschehens in den Blick zu nehmen. So ließe sich fragen, ob die besondere Brutalität der japanischen Armee bei der Einnahme von Nanking, bei der über einen Zeitraum von mehreren Wochen tausende Männer gefoltert und getötet und tausende Frauen und Kinder verstümmelt, vergewaltigt und getötet wurden, mit der situativen Dynamik hinreichend erklärt werden kann. Collins geht davon aus, dass sich im Vorfeld des Massakers bei den japanischen Soldaten über Monate hinweg „Konfrontationsanspannung und -angst“ aufgebaut habe, bis sich durch den Sieg im Kampf um Shanghai ein entscheidendes Überlegenheitsgefühl gegenüber dem Feind eingestellt habe. Die aufgestaute Anspannung habe sich in Nanking in Form einer „Vorwärtspanik“ entladen. Kann sich eine solche emotionale Anspannung tatsächlich über Monate hinweg aufbauen und dann über mehrere Wochen hinweg entladen? Oder hatten vorangegangene Kriegserlebnisse, erlittene und zugefügte Gräueltaten die Soldaten auf eine bestimmte Weise verändert - im Sinne einer Gewöhnung bzw. Abstumpfung gegenüber Gewalt oder anderer „entzivilisierender“ Veränderungen?

Ebenso wäre zu hinterfragen, ob das wochenlange Verstümmeln, Vergewaltigen und Töten bei den japanischen Soldaten nach Abbau der emotionalen Anspannung keine Spuren hinterlassen hat. Während Collins' Gewaltanalysen sich auf situative emotionale Dynamiken konzentrieren, lenkt Elias' Habitus-Konzept den Blick darauf, wie Erfahrungen und Erlebnisse die Psyche (bzw. den Habitus) auch über

16 Siehe dazu die Analysen von Kriegstagebüchern bei Andexlinger und Ebner (2008). 
eine Kampfsituation oder einen Krieg hinaus prägen können. Man denke an die Traumatisierungen und Angststörungen von Kriegsheimkehrern, an den in Soldatentagebüchern beschriebenen Konflikt zwischen zivilen und militärischen sowie zwischen im Training eingeübten und im Grabenkrieg tatsächlich herrschenden Gewaltnormen, oder an andere Probleme, ins Zivilleben zurückzukehren (Kuzmics und Haring 2013, S. 469 ff.).

Zuspitzend könnte man aus einer figurationssoziologischen Perspektive formulieren: Wenn man durch die Art der Fragenformulierung, die Selbstbeschränkung auf eine bestimmte Quellenart und eine entsprechende Fallauswahl von vornherein alles Nicht-Situative aus der Analyse ausschließt, kann die Antwort auf die Frage nach notwendigen Bedingungen nur ,Situation“ lauten. Konstruktiver formuliert, könnte es sich für Forscher*innen, die sich Collins' Gewalttheorie bedienen, lohnen, an folgenden Fragen weiterzuarbeiten: Wie breit kann man eine Situation sinnvollerweise räumlich und zeitlich fassen? Was kann und muss man dabei einbeziehen? Wie spielen langfristige Prägungen und situative Dynamiken zusammen? Und was sind relevante Kontextfaktoren?

\subsection{Collins als Korrektiv für Elias}

Zieht man hingegen Collins als Korrektiv für Elias' Soziologie heran, verdienen vor allem folgende Aspekte Aufmerksamkeit: die Einbeziehung situativer Dynamiken, die Gefahr eines Überstrapazierens des Habitus-Konzeptes und die empirische Überprüfbarkeit langfristiger Prägungen.

\subsubsection{Hinterfragung des Habitus-Reflexes}

Unseres Erachtens könnte Collins' beobachtungsbasiertes Gewaltverständnis dabei helfen, jene Automatismen, Verkürzungen und Fehlkategorisierungen zu vermeiden, für die manche Elias-Epigon*innen besonders anfällig zu sein scheinen. Insbesondere wäre mithilfe der Situationsanalysen das zu hinterfragen, was man etwas polemisch als „Habitus-Reflex“ bezeichnen könnte: die implizite mechanistische Rückführung beobachteter Handlungsmuster oder Eigenschaften auf einen bestimmten schichtspezifischen oder nationalen Habitus. Stattdessen könnte man fragen, welche situativen Dynamiken dabei eine Rolle spielen. Die oben angedeutete Historisierung von „Konfrontationsanspannung“ und „Vorwärtspanik“ könnte hierfür als Beispiel dienen, wobei hier ebenfalls zu fragen wäre, wie langfristige Prägungen und situative Dynamiken jeweils zusammenspielen. Auch wenn von Elias hierzu keine konkreten Überlegungen vorliegen, argumentiert er - anders als manche Anhänger*innen nie kausal-deterministisch. Daran anknüpfend könnte man darüber nachdenken, wie offen verschiedene Habitusschichten, die in unterschiedlichen Sozialisationsphasen entstanden sind, gegenüber situativen Einflüssen sind.

Collins' genaues begriffliches Instrumentarium kann zudem dazu dienen, große prozesssoziologisch-zivilisationstheoretische Erklärungen zu relativieren, die einfache Kausalbeziehungen zwischen bestimmten sozialen Habitus und einer höheren Gewaltneigung herstellen. Mithilfe der Konzepte „Vorwärtspanik“ und „,Moral Holidays" könnte man hinterfragen, ob bestimmte Gräuel tatsächlich auf einen nationalen 
oder ,,modernen“ Habitus zurückzuführen sind, oder ob dafür bestimmte situativemotionale Zwänge verantwortlich sind. Dafür müsste man die Vorstellung nicht aufgeben, dass makrosoziologische Faktoren von Bedeutung sind; man hätte lediglich zu akzeptieren, dass sie die beobachtbaren Variationen in Art, Ausmaß und Intensität von (auch sexueller) Kriegsgewalt nicht hinreichend erklären.

\subsubsection{Vorbeugung moralisierender Verallgemeinerungen}

Vielleicht kann der Hinweis auf Collins' Methode daran erinnern, dass die automatische, verallgemeinernde Rückführung bestimmter Gräueltaten auf einen nicht näher bestimmten nationalen oder schichtspezifischen Habitus mit der Komplexität der eliasschen Argumentation nicht viel gemein hat. Erstens wäre zwischen verschiedenen Graden der Nachhaltigkeit unterschiedlicher Habitusschichten - und entsprechend verschiedenen Arten der Offenheit unterschiedlicher Prägungen gegenüber situativen Einflüssen - zu differenzieren (Kuzmics 2015, S. 227 ff.). Zweitens ist Collins' (2007) Kritik an vielen Anwendungen der ,Trendtheorie“ darin zustimmen, dass sich hinter dem Verweis auf vermeintliche nationale Charaktereigenschaften oft Stereotype verbergen. Eine solche doppelte Relativierung des „Habitus-Reflexes“ scheint vor allem dann angebracht, wenn die ursächliche Rückführung bestimmter Handlungsmuster auf einen sozialen Habitus mit einer moralischen Anklage einhergeht (etwa bei Kriegsvergewaltigungen).

Ferner könnten Elias-Anhänger*innen die Auseinandersetzung mit Collins' Gewalttheorie zum Anlass nehmen, sich mit Einwänden auseinanderzusetzen, die immer wieder gegen sie vorgebracht werden. Sie könnten sich bspw. fragen, inwiefern der Teleologie- oder der Eurozentrismus-Vorwurf berechtigt ist. Trifft er nur auf vereinfachende Anwendungen und Vulgärinterpretation zu oder lassen sich auch legitime Vorbehalte gegen Elias selbst formulieren? Ebenfalls wenig diskutiert ist, dass sich bei Elias mindestens zwei Zivilisations-Begriffe finden, deren einer normative Züge aufweist (Anders 2000; Duerr 1988; Hinz 2002).

\subsubsection{Verweis auf das Problem der empirischen Überprüfbarkeit langfristiger Prägungen}

Collins' rekonstruierende, materialnahe Methode verweist auf ein weiteres grundsätzliches Problem der prozesstheoretisch-historischen Soziologie, das allgemein mehr Aufmerksamkeit verdienen würde: die Frage der empirischen Überprüfbarkeit langfristiger habitueller Prägungen. Dies hängt nicht nur damit zusammen, dass aufgrund fehlender Quellen - langfristige kollektive Handlungsmuster oder Persönlichkeitszüge (über Jahrhunderte) nur schwer nachzuweisen sind.

Vielmehr warten noch grundlegendere Fragen darauf, diskutiert zu werden: Lassen sich für die Mitglieder gesellschaftlicher Großgruppen (Fürstentümer, Staaten, Schichten) tatsächlich kollektiv geteilte Persönlichkeitszüge und Verhaltensmuster empirisch nachweisen, die sich über längere Zeit von den Charaktereigenschaften der Mitglieder anderer Großgruppen unterscheiden? Wie kann man diese Habitus und deren Wandel - mittels belastbarer Quellen mit bestimmten gesellschaftlichen Strukturen - und deren Wandel - in Verbindung bringen? Und wie lässt sich ent- 
scheiden, ob diese gruppentypischen Eigenschaften das Ergebnis langfristiger Prägungen durch relativ stabile Institutionen und Sozialisationsinstanzen (Ehe, Schule, Medien, Bürokratie, Militär) sind, oder ob diese relativ stabilen Institutionen lediglich immer wieder ähnliche Situationen hervorbringen, die dann ihrerseits situativ handlungswirksam werden?

So könnte man die gegenwärtige Abneigung der meisten Mitteleuropäer*innen gegenüber körperlicher Gewalt entweder als Ausdruck eines sozial erlernten, relativ zivilisierten, mit umfassenden emotionalen Selbstzwängen ausgestatteten, Habitus verstehen, der der relativen Friedlichkeit der inner- und zwischenstaatlichen Beziehungen der meisten mitteleuropäischen Staaten der letzten Jahrzehnte entspricht; oder aber als Ergebnis der Tatsache, dass von fremdbestimmenden Settings - wie dem staatlichen Gewaltmonopol, dem engmaschigen Netz zwischenstaatlicher Beziehungen oder der vorherrschenden öffentlichen Meinung - ständig situative Zwänge ausgehen, die die Akteur*innen davon abhalten, nicht-spielerische physische Gewalt (außer in dafür vorgesehenen Enklaven) auszuüben, anzusehen oder gutzuheißen.

\section{Bilanz: Was (und wem) nützt der Dialog?}

In diesem Artikel haben wir zu zeigen versucht, dass sowohl die mikro- als auch die figurationssoziologische Gewaltforschung davon profitieren würde, wenn sie ihre eigenen Axiome aus der Perspektive des jeweils anderen Ansatzes betrachten und dessen Einwände ernst nehmen würden. Wir haben argumentiert, dass es sich gerade - aber bei weitem nicht nur - für die Untersuchung eines theoretisch hochkomplexen, empirisch schwer zugänglichen und für normative Wertungen besonders anfälligen Gegenstandes wie sexuelle Kriegsgewalt lohnen könnte, eingefahrene Denkvorstellungen zu hinterfragen. Wie sich zeigte, können nicht nur potenzielle Gemeinsamkeiten und Überschneidungen, sondern auch Unterschiede und Widersprüche produktiv sein. Abschließend bleibt zu fragen, was die Soziologien collinsscher und eliasscher Provenienz dazu beitragen können, die im ersten Abschnitt angeführten Mängel der vorliegenden Literatur zu sexueller Kriegsgewalt zu überwinden.

Erstens ist zu fragen, inwiefern die beiden Ansätze als Korrektiv für problematische Schwerpunkte der Forschung dienen können. Collins und Elias wirken der Normativität der medialen, politischen und wissenschaftlichen Debatte insofern entgegen, als sie explizit anstreben, zum besseren Verständnis von Gewalt(-bereitschaft) beizutragen. Obwohl beide emotionale Wertungen, Wiedergutmachungsansprüche und Verurteilungsforderungen für nachvollziehbar halten, betonen sie, dass eine normativ-engagierte Sichtweise dem Verständnis - und damit auf lange Sicht auch der Vorbeugung - von Gewalt im Wege steht. Mit Blick auf den selbstbewussten Generalisierungsanspruch kann man einerseits festhalten, dass beide Soziologien dem Essentialismus biologistischer und feministischer Ansätze entgegenstehen. Andererseits würde es auch ihnen guttun, ihre eigenen Allgemeinheitsansprüche zu überprüfen. Collins' und Elias' Betonung des Stellenwerts emotionaler Dynamiken bei der Ausübung von physischer Gewalt bietet nicht nur wichtige Argumente gegen 
die rationalistisch-voluntaristische Kriegsstrategie-These, sie eröffnet auch spannende Fragen zum Verhältnis von Emotion und Kognition.

Zweitens haben wir zu zeigen versucht, dass Situations- und Figurationsanalysen dazu beitragen können, die eingangs angeführten blinden Flecken der Literatur zu beleuchten.

Und drittens können Collins' beobachtungsnahes Vokabular und Elias' wissenssoziologisch begründete Begriffsbildung einen Beitrag zur sprachlichen Präzisierung und zur Versachlichung leisten. Ein Ausweg aus der aktuellen Begriffsverwirrung könnte darin bestehen, eingefahrene Begriffe, die - ob beabsichtigt oder nicht - bestimmte Konnotationen mit sich bringen und so das Denken in die eine oder andere Richtung drängen, zu vermeiden, und stattdessen neue Begriffe induktiv aus dem empirischen Material zu entwickeln. Unseres Erachtens bietet sich dazu die Methode der Rekonstruktion an, die Collins z.B. zur Untersuchung der Kriegsgräuel von Nanking angewandt hat. Dabei werden die detailliert rekonstruierten Handlungs- und Interaktionsabläufe schrittweise abstrahiert und zusammengefasst. Diese Methode ist einerseits offen genug, um verschiedenste Quellenarten einzubeziehen, was angesichts der dürftigen Quellenlage notwendig ist. Anderseits ermöglicht sie, das Material einzuordnen, zu strukturieren, zu interpretieren und zu einem möglichst kohärenten Bild des Gewaltverlaufes zusammenzufügen. Auf dieser Basis würde die (auch ideologische) Entweder-Oder-Frage, ob eine Vergewaltigung als gewaltsame Ausübung sexueller Handlungen oder als sexuelle Ausübung männlicher Macht zu verstehen sei, in eine Frage verwandelt, die man jeweils empirisch beantworten und gleichzeitig zur Arbeit an immer robusteren Begriffen und theoretischen Modellen nutzen kann: Unter welchen situativen und/oder figurativen Umständen sind welche Erscheinungsformen der sexuellen und nicht-sexuellen Gewalt bzw. der gewaltsamen und nicht-gewaltsamen Sexualität zu beobachten?

Gleichzeitig stellt sich die Frage nach den Kosten, die für die beiden Ansätze aus einer Verknüpfung resultieren könnten. Das hier vorgelegte Ansinnen muss sich insbesondere dem Einwand stellen, der Komplexität der einzelnen Perspektiven nicht gerecht zu werden. Konkret birgt der Syntheseversuch drei Gefahren: erstens, dass einzelne Begriffe verwässern, statt geschärft und präzisiert zu werden; zweitens, dass das Ergebnis den Axiomen der Ansätze so grundsätzlich widerspricht, dass es ihnen nicht genügt; und drittens, dass das Ergebnis ein sehr allgemeiner Kompromiss ist, der wenig Erhellendes beizutragen hat, das über einen der beiden Ansätze (bzw. eine getrennte Beschäftigung mit beiden Ansätzen) hinausgeht.

In Anbetracht dessen sei darauf hingewiesen, dass wir nicht dafür plädieren, einzelne Konzepte aus dem jeweiligen Gedankengebäude zu lösen und beliebig mit Konzepten aus der jeweils anderen Theorietradition zu kombinieren. Vielmehr schlagen wir vor, die jeweils andere Perspektive zunächst als Korrektiv für die eigene Position anzusehen. Wenn sich dies als fruchtbar herausstellt, kann man fragen, ob sich einzelne Konzepte oder Einsichten des anderen Ansatzes in den eigenen integrieren lassen - immer vor dem Hintergrund der Frage, ob und wie diese miteinander zu vereinbaren sind und wie sich eine Zusammenführung auf das ganzes Gedankengebäude auswirkt. Aber auch der umgekehrte Versuch, ausgewählte eigene Konzepte in das entgegengesetzte Theoriegebäude einzubauen, mag ein lohnendes 
Gedankenexperiment sein, das dazu beitragen kann, für selbstverständlich gehaltene Überzeugungen in Frage zu stellen.

Dabei hat sich zweierlei gezeigt: Erstens, dass es bereits exemplarische Überlegungen erlauben, überraschende Querbezüge herzustellen und den eigenen wie den anderen Ansatz in einem anderen Licht zu sehen; und zweitens, dass diese kleinen Fortschritte tatsächlich ständig durch die Gefahr der Banalisierung auf der einen Seite und der einseitigen Verzerrung auf der anderen Seite bedroht sind. Diese Risiken sind unseres Erachtens gerechtfertigt, da die synthetisierende und kontrastierende Vorgehensweise umgekehrt dazu beitragen kann, andere, nicht minder problematische Verkürzungen der einen oder anderen gewaltsoziologischen Theorie zu überwinden, die zu Fehlkategorisierungen, Perspektiveinengungen und Pauschalisierungen führen können. Die damit verbundene Hinterfragung eingefahrener Sprach- und Denkgewohnheiten scheint gerade im Falle eines ideologisch aufgeladenen, theoretisch komplexen und empirisch schwer zugänglichen Phänomens wie sexueller Kriegsgewalt durchaus geboten.

Gibt man, wenn auch nur vorübergehend, bestimmte Dogmen und universelle Geltungsansprüche auf, erscheint es sowohl möglich die breite figurationssoziologische Perspektive durch die Untersuchung situativer Dynamiken anzureichern als auch prozesssoziologische Erkenntnisse in mikrosoziologische Situationsanalysen einzubeziehen. Die Autor*innen dieses Beitrages arbeiten derzeit getrennt voneinander an solchen integrierenden Ansätzen.

Funding Open Access funding provided by Projekt DEAL.

Open Access Dieser Artikel wird unter der Creative Commons Namensnennung 4.0 International Lizenz veröffentlicht, welche die Nutzung, Vervielfältigung, Bearbeitung, Verbreitung und Wiedergabe in jeglichem Medium und Format erlaubt, sofern Sie den/die ursprünglichen Autor(en) und die Quelle ordnungsgemäß nennen, einen Link zur Creative Commons Lizenz beifügen und angeben, ob Änderungen vorgenommen wurden.

Die in diesem Artikel enthaltenen Bilder und sonstiges Drittmaterial unterliegen ebenfalls der genannten Creative Commons Lizenz, sofern sich aus der Abbildungslegende nichts anderes ergibt. Sofern das betreffende Material nicht unter der genannten Creative Commons Lizenz steht und die betreffende Handlung nicht nach gesetzlichen Vorschriften erlaubt ist, ist für die oben aufgeführten Weiterverwendungen des Materials die Einwilligung des jeweiligen Rechteinhabers einzuholen.

Weitere Details zur Lizenz entnehmen Sie bitte der Lizenzinformation auf http://creativecommons.org/ licenses/by/4.0/deed.de.

\section{Literatur}

Anders, Kenneth. 2000. Die unvermeidliche Universalgeschichte. Studien über Norbert Elias und das Teleologieproblem. Opladen: Leske + Budrich.

Andexlinger, Silvia, und Johannes Ebner. 2008. „Friedlich leuchtet die Sonne auf Tod und Leben“. Die Erfahrung des Ersten Weltkrieges in literarischen und nicht-literarischen Quellen. In Das Gesicht des Krieges: Militär aus emotionssoziologischer Sicht Schriftenreihe der Landesverteidigungsakademie., Hrsg. Sabine A. Haring, Helmut Kuzmics, 59-113. Wien: Landesverteidigungsakademie.

Beck, Birgit. 2004. Wehrmacht und sexuelle Gewalt. Sexualverbrechen vor deutschen Militärgerichten 1939-1945. Paderborn: Ferdinand Schöning.

Brownmiller, Susan. 1980. Gegen unseren Willen. Vergewaltigung und Männerherrschaft. Frankfurt a.M.: Fischer. 
Clark, Janine Natalaya. 2014. A crime of identity: rape and its neglected victims. Journal of Human Rights 13:146-169.

Cohen, Dara Kay, und Ragnhild Nordås. 2014. Sexual violence in armed conflict: Introducing the SVAC dataset, 1989-2009. Journal of Peace Research 51:418-428.

Collins, Randall. 2007. A dead end for a trend theory. Cambridge: Polity Press.

Collins, Randall. 2008. Violence. A micro-sociological theory. Princeton: Princeton University Press.

Collins, Randall. 2009. Micro and macro causes of violence. International Journal of Conflict and Violence 3:9-22.

Collins, Randall. 2011a. Dynamik der Gewalt. Eine mikrosoziologische Studie. Hamburg: Hamburger Edition.

Collins, Randall. 2011b. Four theories of informalization and how to test them. In Cultuur en ongelijkheid, Hrsg. Christien Brinkgreve, Marcel van den Haak, Bart van Heerikhuizen, Johan Heilbron, und Giselinde Kuipers, 160-172. Diemen: AMB Press.

Collins, Randall. 2014. Four theories of Informalization and how to test them. human figurations: longterm perspectives on the human condition 3. http://hdl.handle.net/2027/spo.11217607.0003.207. Zugegriffen: 19. Okt. 2017.

Collins, Randall. 2015. Vorwärtspaniken und die Dynamik von Massengewalt. In Gewaltmassen. Über Eigendynamik und Selbstorganisation kollektiver Gewalt, Hrsg. Axel T. Paul, Benjamin Schwalb, 204-230. Hamburg: Hamburger Edition.

Collins, Randall. 2019. An der Schwelle zur Gewalt. Ein Gespräch mit Randall Collins. Mittelweg 36(28):60-69.

Colllins, Randall. 1999. Macrohistory. Essays in sociology of the long run. Stanford: Stanford University Press.

Cooney, Mark. 2009. The scientific significance of collins's violence. The British Journal of Sociology 60:586-594.

Duerr, Hans Peter. 1988. Nacktheit und Scham. Der Mythos vom Zivilisationsprozeß. Bd. 1. Frankfurt a.M.: Suhrkamp.

Ebner, Johannes. 2018. Tradition ohne Vergangenheit. Zur sozialen Neudefinition von alpinen Maskenbräuchen. Wiesbaden: Springer VS.

Elias, Norbert. 1992. Studien über die Deutschen. Machtkämpfe und Habitusentwicklungen im 19. und 20. Jahrhundert. Frankfurt a.M.: Suhrkamp.

Elias, Norbert. 1997. Über den Prozeß der Zivilisation. Soziogenetische und psychogenetische Untersuchungen. Frankfurt a.M.: Suhrkamp. 2 Bände.

Elias, Norbert. 2001. Die Gesellschaft der Individuen. Frankfurt a.M.: Suhrkamp.

Elias, Norbert. 2003a. Engagement und Distanzierung. In Gesammelte Schriften 8, 105-169. Frankfurt a.M.: Suhrkamp.

Elias, Norbert. 2003b. Gedanken über die große Evolution. In Gesammelte Schriften 8, 285-366. Frankfurt a.M.: Suhrkamp.

Elias, Norbert. 2003c. Die Fischer im Mahlstrom. In Gesammelte Schriften 8, 171-281. Frankfurt a.M.: Suhrkamp.

Elias, Norbert. 2006a. Zivilisation und Gewalt. In Gesammelte Schriften 15, 72-117. Frankfurt a.M.: Suhrkamp.

Elias, Norbert. 2006b. Wandlungen der Machtbalance zwischen den Geschlechtern. Eine prozeßsoziologische Untersuchung am Beispiel des Antiken Römerstaats. In Gesammelte Schriften 15, 139-234. Frankfurt a.M.: Suhrkamp.

Elias, Norbert. 2006c. Über den Rückzug der Soziologen auf die Gegenwart I. In Gesammelte Schriften 15, 389-408. Frankfurt a.M.: Suhrkamp.

Elias, Norbert. 2006d. Figuration, sozialer Prozess und Zivilisation: Grundbegriffe der Soziologie. In Gesammelte Schriften 16, 100-117. Frankfurt a.M.: Suhrkamp.

Elias, Norbert. 2006e. Technisierung und Zivilisation. Beitrag für den Deutschen Soziologentag am 30.9.1986. In Gesammelte Schriften 16, 182-234. Frankfurt a.M.: Suhrkamp.

Elias, Norbert. 2006f. Was ich unter Zivilisation verstehe. Antwort auf Hans Peter Duerr. In Gesammelte Schriften 16, 334-341. Frankfurt a.M.: Suhrkamp.

Elias, Norbert, und John L. Scotson. 1993. Etablierte und Außenseiter. Frankfurt a.M.: Suhrkamp.

Eriksson Baaz, Maria, und Maria Stern. 2010. The complexity of violence: a critical analysis of sexual violence in the democratic republic of Congo (DRC). Working paper on gender based violence. Stockholm: The Nordic Afrika Institute.

Eriksson Baaz, Maria, und Maria Stern. 2013. Sexual violence as a weapon of war? Perceptions, prescriptions, problems in the Congo and beyond. London: Zed Books. 
Galtung, Johan. 1975. Strukturelle Gewalt. Reinbek: Rowohlt.

Gebhardt, Miriam. 2015. Als die Soldaten kamen. Die Vergewaltigung deutscher Frauen am Ende des Zweiten Weltkriegs. München: DVA.

Goldstein, Joshua. 2001. War and gender. How gender shapes the war system and vice versa. Cambridge: Cambridge University Press.

Górnicka, Barbara. 2016. Nakedness, shame, and embarrassment. A long-term sociological perspective. Wiesbaden: Springer VS.

Henry, Nicola, Tony Ward, und Matt Hirshberg. 2004. A multifactorial model of wartime rape. Aggression and Violent Behavior 9:535-562.

Hinz, Michael. 2002. Der Zivilisationsprozess: Mythos oder Realität? Wissenschaftssoziologische Untersuchungen zur Elias-Duerr-Kontroverse. Opladen: Leske + Budrich.

Hoebel, Thomas, und Wolfgang Knöbl. 2019. Gewalt erklären! Plädoyer für eine entdeckende Prozesssoziologie. Hamburg: Hamburger Edition.

Hoebel, Thomas, und Stefan Malthaner (Hrsg.). 2019. Im Brennglas der Situation. Neue Ansätze in der Gewaltsoziologie. Mittelweg 3628 (1/2).

Hüttermann, Jörg. 2012. Zeit, Raum und Macht: Eine figurationssoziologische Meditation über Grundbegriffe der Soziologie. Unveröffentlichtes Manuskript.

Hüttermann, Jörg, und Johannes Ebner. 2020. Von der Einfalt der Turns zur Vielfalt der Sachorientierung: Anmerkungen zur deutschen Gewaltforschung im Lichte aktueller Publikationen. Soziologische Revue: im Erscheinen.

Isikozlu, Elvan. 2011. Kriegsvergewaltigung. Eine Typologie. Wissenschaft und Frieden 67:1-12.

Joas, Hans, und Wolfgang Knöbl. 2004. Sozialtheorie: Zwanzig einführende Vorlesungen. Frankfurt a.M.: Suhrkamp.

Kelly, Jocelyn. 2010. Rape in war: motives of militia in DRC. United States Institute of Peace. Special Report 243.

Kirby, Paul. 2012. How is rape a weapon of war? Feminist international relations, modes of critical explanation and the study of wartime sexual violence. European Journal of International Relations 19:797-821.

Kuzmics, Helmut. 2015. Habitus versus Situation. Elias' und Collins’ Erklärungen von Gewalt und Gewaltbereitschaft im Krieg am Beispiel eines habsburgischen Militärhabitus im Ersten Weltkrieg. In Wo denken wir hin? Lebensthemen, Zivilisationsprozesse, demokratische Verantwortung, Hrsg. HansPeter Waldhoff, Christine Morgenroth, Angela Moré, und Michael Kopel, 215-236. Gießen: Psychosozial-Verlag.

Kuzmics, Helmut, und Sabine A. Haring. 2013. Emotion, Habitus und Erster Weltkrieg. Göttingen: Vandenhoek \& Ruprecht.

Lilly, J. Robert. 2007. Taken by force. Rape and American gis in Europe during world war II. New York: Palcrave Macmillan.

Linklater, Andrew. 2017. Violence and civilization in the western states-systems. Cambridge: Cambridge University Press.

Mennell, Stephen. 2007. The American civilizing process. Cambridge: Polity Press.

Nungesser, Frithjof. 2014. Individuation und Ekstase. Kollektive Erregung als subjekttheoretischer Stachel und intersubjektivitätstheoretische Herausforderung. In Vielfalt und Zusammenhalt. Verhandlungen des 36. Kongresses der DGS in Bochum und Dortmund 2012., Hrsg. Martina Löw, 785-796. Frankfurt a.M.: Campus.

Pinker, Steven. 2011. Gewalt. Eine neue Geschichte der Menschheit. Frankfurt a.M.: Fischer.

Rössel, Jörg. 2012. Randall Collins. Konflikttheorie. Ausgewählte Schriften. Wiesbaden: Springer VS.

Scheff, Thomas J. 1994. Bloody revenge: emotions, nationalism and war. Boulder: Westview Press.

Scheff, Thomas J., und Suzanne M. Retzinger. 1997. Shame, anger and the social bond: a theory of sexual offenders and treatment. Electronic Journal of Sociology. https://www.sociology.org/ejs-archives/ vol003.001/sheff.html. Zugegriffen: 19. Okt. 2017.

Skjelsbæk, Inger. 2001. Sexual violence and war: mapping out a complex relationship. European Journal of International Relations 7:211-237.

Skjelsbæk, Inger. 2015. The military perpetrator: a narrative analysis of sentencing judgments on sexual violence offenders at the international criminal tribunal for the former yugoslavia (ICTY). Journal of Social and Political Psychology 3:46-70.

Stopfinger, Marion. 2016. Kriegsvergewaltigung - Eine kritische Auseinandersetzung mit der feministischen Perspektive von Susan Brownmiller am Beispiel des Zweiten Weltkrieges. Masterarbeit. Graz: Institut für Soziologie, Karl-Franzens-Universität Graz. 
Sutterlüty, Ferdinand. 2015. Kollektive Gewalt und urbane Riots. Was erklärt die Situation. In Über Eigendynamik und Selbstorganisation kollektiver Gewaltmassen, Hrsg. Axel T. Paul, Benjamin Schwalb, 231-256. Hamburg: Hamburger Edition.

Sutterlüty, Ferdinand. 2017. Fallstricke situationistischer Gewaltforschung. WestEnd. Neue Zeitschrift für Sozialforschung 14:139-155.

Sutterlüty, Ferdinand, Matthias Jung, und Andy Reymann (Hrsg.). 2019. Narrative der Gewalt. Interdisziplinäre Analysen. Frankfurt a.M.: Campus.

de Swaan, Abram. 2015. The killing compartments. The mentality of mass murder. New Haven: Yale University Press.

von Trotha, Trutz. 1997. Zur Soziologie der Gewalt. Kölner Zeitschrift für Soziologie und Sozialpsychologie 49 (Sonderheft 37):9-56.

Wood, Elisabeth Jean. 2006. Variation in sexual violence during war. Politics \& Society 34:307-341.

Wood, Elisabeth Jean. 2009. Armed groups and sexual violence: when is wartime rape rare? Politics \& Society 37:131-162.

Wood, Elisabeth Jean. 2014. Conflict-related sexual violence and the policy implications of recent research. International Review of the Red Cross 96:457-478.

Wouters, Cas. 2004. Sex and Manners: Female Emancipation in the West 1890-2000. London: SAGE.

Wouters, Cas. 2007. Informalization: manners and emotions since 1890. London: SAGE.

Wouters, Cas, und Stephen Mennell. 2015. Discussing theories and processes of Civilisation and Informalisation: Criteriology. human figurations: long-term perspectives on the human condition 4. http:// hdl.handle.net/2027/spo.11217607.0004.302. Zugegriffen: 19. Okt. 2017.

Johannes Ebner studierte u. a. Soziologie in Graz. 2011-2012 war er Wissenschaftlicher Mitarbeiter an der TU Graz, 2014-2018 Universitätsassistent und Lektor am Institut für Soziologie der Universität Graz. Seit Mai 2018 ist er Wissenschaftlicher Mitarbeiter am Institut für Interdisziplinäre Konflikt- und Gewaltforschung (IKG) der Universität Bielefeld. Zu seinen Arbeitsschwerpunkten gehören die figurationssoziologische Konflikt- und Gewaltforschung, die Zivilisations- und Prozesstheorie sowie die ethnographische Sozialraumforschung.

Marion Stopfinger studierte u. a. Soziologie in Graz. Sie war 2015 Studentische Mitarbeiterin am Institut für Soziologie der Universität Graz und erhielt 2016 ein Shortcuts-Stipendium an der Universität Bielefeld (Thema: Die Kriegsvergewaltigungen während des Bosnienkrieges - Strategie oder „Vorwärtspanik“?). Derzeit lehrt sie u. a. zu diesem Thema an der Universität Graz. Zu ihren Forschungsschwerpunkten gehören Krieg und Gewalt und im Speziellen sexuelle Kriegsgewalt. 